\title{
Design of Active Holonic Fault-Tolerant Control Systems
}

\author{
Robson M. da Silva ${ }^{1}$, Paulo E. Miyagi ${ }^{2}$, and Diolino J. Santos Filho ${ }^{2}$ \\ ${ }^{1}$ State University of Santa Cruz, Rod. Ilhéus/Itabuna, km 16, CEP 45662-900 Ilhéus, BA \\ ${ }^{2}$ University of São Paulo, Av. Prof. Mello Moraes, 2231, CEP 05508-030 São Paulo, SP \\ Brazil \\ rmsilva@uesc.br, pemiyagi@usp.br, diolino.santos@poli.usp.br
}

\begin{abstract}
The adequate evaluation of new technologies in productive systems that perform multiple and simultaneous processes, exploring the intense sharing of resources, demands the updating of supervision and control systems. On the other hand, totally infallible systems are unviable, and for a flexible productive system (FPS) does not to suffer interruptions due to component failure, the concept of AFTCS (active fault-tolerant control system) mechanism must be adopted. In this sense, holonic control system (HCS) is considered a trend for an intelligent automation and the combination of HCS techniques and AFTCS is fundamental to assure efficiency, flexibility and robustness of FPSs. Therefore, this work presents a procedure for the modeling of active holonic fault-tolerant control system (AHFTCS) that considers AFTCS' requirements and interpreted Petri net for the description of the systems' behavior. This method is applied to an intelligent building (IB) as a class of FPS and the results are presented.
\end{abstract}

Keywords: control system, system modeling, fault-tolerance, Petri net, holon, reconfiguration.

\section{Introduction}

Advances of mechatronic systems, communication networks and work organization methods, allied to the crescent competitiveness and the need for efficient services triggered great changes on productive systems (PSs) requiring more flexibility under different demands, such as production volume, type of product and nature of resources involved. The flexible productive systems (FPSs) were designed to attend the current production demands and the focus is in material technological transforming, the information processing and service execution. Therefore manufacturing systems as well as intelligent buildings (IBs) can be approached as FPS. These systems perform multiple and simultaneous processes, exploring the intense sharing of resources, which makes complex the supervision and control of the systems global behavior [1-3].

The supervision and control systems have been evolved from a centralized and hierarchic architecture to a heterarchical and distributed architecture. This distributed system (DS) is composed of various sub-systems (that can be physically installed at 
different geographical locations), in which the tasks are divided according to the required functionality and processing capacity of each equipment [3]. On the other hand, to assure that a FPS does not suffer interruption due to faults of its components, an AFTCS (active fault-tolerant control system) mechanism must be considered [4]. This mechanism involve the detection of the fault, study of its effects, identification of causes and finally, the system reconfiguration that is done by relocating processes and choosing alternative interaction paths between processes [5]. In case of faults the strategy is to recover the system functionalities (regeneration) or to maintain critical operations in such a way that some parts of the system are disabled, but not affecting other parts of the system (degeneration).

In this context, the integration of MAS (multi-agent system) and HS (holonic system) techniques with mechatronic technology, called holonic control system (HCS), is considered a trend for the intelligent automation of PSs $[1,3,6,7]$. The aim is to explore MAS and HS concepts of superposition, such as autonomy, reactivity, proactivity, cooperation, social capacity (i.e., consideration of the human interaction on processes), and learning resources; and to take advantage from the complementary features in the implementation of HSs by means of the MASs.

However, most of supervision and control systems do not adopt HCS and AFTCS mechanisms. In fact, the amount of material published about modeling of processes that consider the use of these techniques is very little $[1,4,6]$. Therefore, this research presents a procedure for modeling and operation of active holonic fault-tolerant control system (AHFTCS) considering a sustainability approach and its functional specifications in normal circumstances and also during faults. The application of a HVAC (heating, ventilation and air conditioning) subsystem of an intelligent building (IB) [8] is presented to illustrate the advantages of the procedure.

\section{Contribution to Technological Innovation for Sustainability}

The new strategic approach "sustainability research" addresses the three conflicting aspects: contributing to economic development, being ecologically acceptable and socially just [9]. It is expected that this approach will lead to more sustainable solutions and thus more effective and efficient spending of public and private funds for research and development. To live "sustainability" and make this a brand for the 21 st century requires a strong engagement of science, industry and politics. Priority topics of relevance suggested by the participants for the German-Brazilian cooperation on science for sustainability were highlighted during the discussion and included: renewable energy, transportation and logistics, environmental technologies, sustainability in buildings, especially governmental buildings and industrial plants, and others $[9,10]$.

In this context, the rational use of energy, minimization of operational costs, larger safety and comfort to the users are essential characteristics in intelligent buildings (IBs). But as mentioned before, IB can be approached as a case of FPS. That is, this work is also a contribution for the technological innovation in design methods of IBs.

Considering also previous works in the area of IBs and FPSs, here we adopt the approach of discrete event system (DES) [3, 8], i.e, Petri net (PN) and its extensions is used for description of the system behavior (its productive processes). If compared to 
other description techniques of DES, PN has an equivalent modeling power and it also has the characteristic and advantage of system visualization $[11,12]$.

A survey $[1-4,6,7,15]$ shows that: i) there is a small number of works that consider the integration of HCS and AFTCS requirements; ii) there are few practical applications for these agent technologies, showing that there is still a long way to go to spread these HSs, iii) in most of these systems, there is no negotiation mechanism between holons, iv) there is no information about the use of a systematic method to structure and rationalize the proposed development models, since phase of specifications until operation one, such as PN models can be used.

Therefore, to model the dynamic behavior of FPS, a place/transition Petri net class was adopted, herein called extended Petri net (e-PN), to which temporized transitions, inhibitor arcs and enabling arcs (terms related to PN are in Arial) were added [11]. To systematize and make easier the modeling these models a channel/agent PN type called PFS (Production Flow Schema) [11] is used. The system's dynamic models are generated by means of e-PN. Thus, the procedure combines the bottom-up approach and the top-down approach of the stepwise refinement associated to PFS.

According to Fig.1a, the procedure presents mechanisms that allow the switching of the control in two modes: hierarchic and heterarchic control architecture. It allows the switching of control between two operational modes: the "stationary mode" where the control system is coordinated in a hierarchical; and the "transient mode", where to assure more system flexibility and agile behavior. This architecture is described in Section 3 .

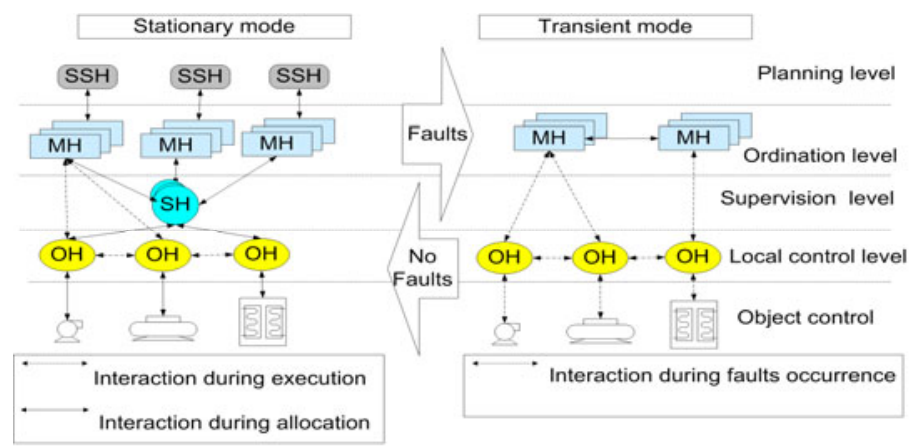

Fig. 1. Control Architecture of AHFTCS

The procedure adapts what is presented in [13] to specification of a mechanism called "diagnoser" using an e-PN model. Toward decision making phase of the AFTCS, some inference rules based on reasoning [14] may be adopted for the specification of a mechanism called "decider". Besides, a system that considers the reconfiguration requires redundant resources to keep an adequate performance, and must also consider the transmission of control signals as part of the system to be controlled, because a fault on this communication network may also limit the coherence of command actions [4, 15]. 


\section{Procedure for AHFTCS Design}

Here the basic structure of the AHFTCS development procedure is presented: analysis of requirements, modeling, analyzing/simulation, implementation and operation. In the following explanation of each phase of the procedure it is presented some examples of models derived from case study applied. In Fig. 2a, is presented a cold production subsystem from a commercial building in São Paulo city, Brazil, composed of two chillers, four pumps, two block valves and one flowmeter (F).

Phase 1 - analysis of requirements - on this phase is defined the AHFTCS' specifications: aim of the system, control object, control devices, definition of tasks, strategies and control functions, and description of the interaction between the parts of the system, and the cases of reconfiguration.

Sub-phase 1.1 - identification of holons - on this sub-phase the holons are identified, i.e., SSH, MH, SH and $\mathrm{OH}$. The holarchies are represented by ellipsis and one holon may belong, simultaneously, to various holarchies (Fig. 2b). The identification of SSH - subsystem holon - involves the definition of control functions of each product/service offered by the FPS' subsystems and how to perform production/service orders. Thus, SSH contains all knowledge necessary to operate the FPS and to choose the better strategy to reach the objectives planned. The MHs manager holons - are the entities responsible for the management of control strategies that must be followed during execution phase. The SHs are responsible for coordinating the OHs. The SH - supervisor holon - contains all knowledge necessary to coordinate holons on lower hierarchic levels. The function of the SH involves the preparation of a program of tasks and coordination of decisions for the performance of these tasks. When a process requests a resource, in fact it is requesting functionality and the SHs check the available resources to control the allocation of the resource. The $\mathrm{OH}$ - operational holon - represents human operators and plant's physical resources, which have any control device for its operation and establish these resources' behavior according to the objectives and skills. $\mathrm{OH}$ manages the behavior of these resources according to the objectives, characteristics and skills. According to Fig. 2, a holarchy (CP) is formed by the $\mathrm{SH}$ cold production controller (SH CP) and other holarchies: main production (MP), auxiliary production (AP) and distribution (Dist) and these holarchies are represented at other SH and OHs. For this subsystem, which provide cold water to the heat exchanger of the air conditioning unit; the control actions are developed considering redundancy: the activity [production of cold water] may be carried out in the main cold subsystem, in the auxiliary cold subsystem, or both.

Sub-phase 1.2 - AFTCSs specifications - in this sub-phase is identified the main critical points of the system and the faults that may affect the normal performance of functions indispensable to the system. After that identification it is necessary to analyze which critical processes will be subject to reconfiguration. The functions of the AFTCS are divided into four phases and are present at each holon independently of the type. The "estimation" phase involves: 1) detection of symptoms that may supervise the existence of faults and 2) the isolation of the fault. When the symptoms detected do not allow any conclusion, the system must be programmed to identify the kind of fault detected in similar cases or request external intervention. The "planning" phase decides upon the reconfiguration action based on pre-defined priorities such as: 
lower performance fall, lower recovery time, etc. The "execution" phase involves the sending of commands for the performance of the selected action plan. The last phase is "learning", which involves the storage of the relevant data in relation to the performed plan. Therefore, it may be stated that AHFTCS acts according to the following AFTCS rules: if <symptoms $>$ then $<$ selects fault $>$; if $<$ fault selected $>$ then $<$ selects action $>$; if $<$ action selected $>$ then $<$ activates reconfiguration $>$; and if $<$ reconfiguration performed $>$ then $<$ storage relevant data $>$.

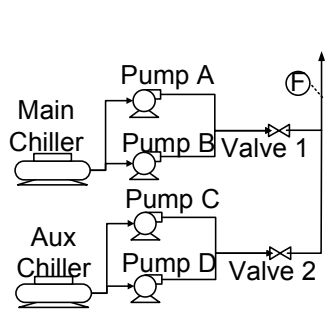

(a)

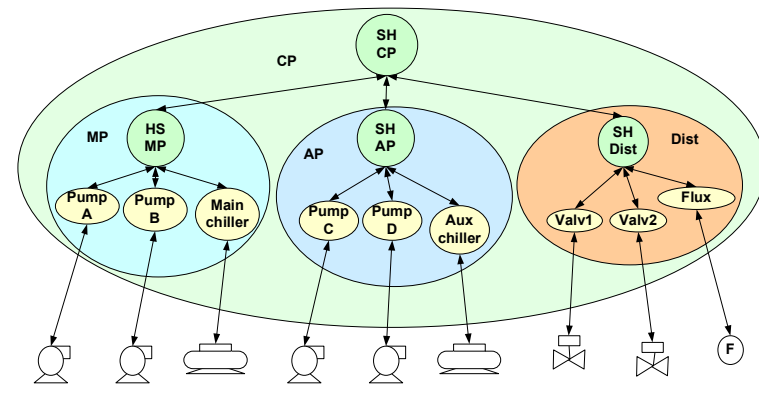

(b)

Fig. 2. (a) Cold production sub-system and (b) organization holarchies

Sub-phase 1.3 - definition of interaction patterns between holons - three interactive processes are considered in this sub-phase: "request for products/services", "execution" of products/services, "fault treatment" and "reconfiguration" due to faults. The synchronization of e-PN models is made by enabling arcs and inhibitors (Fig. 3). These interactions are extracted from UML sequence diagram [16].

Phase 2 - modeling considering reconfiguration - using PFS models represents the interactions of negotiation between holons, and the submission of orders to operational holons OHs; preparation and performance of these orders; and the treatment of faults upon their occurrence. The occurrence of faults must be represented by means of SHs and OHs' models. The control strategies of the AFTCS are modeled on this phase, with the "diagnoser" and the "decider" to fulfill the requirements of the diagnosis and decision phases. The steps to design the e-PN model of the "diagnoser" are: i) construction of e-PN models for the components of the control object; ii) construction of e-PN models of control strategies; iii) definition of observable events - generally those related to control strategy commands; and nonobservable events [13], generally related to faults; iv) construction of e-PN models of sensors; v) initiate the construction of the "diagnoser" from the initial state considered "normal" (without faults); vi) relate, by means of transitions and enabling arches, the performed strategies with possible observable and non-observable events which may happen from the initial state; and vii) relate the states obtained with the states of the sensors. If the "diagnoser" does not indicate the correct state then the possible faults' causes must be inferred to solve possible conflicts. This decision mechanism is called "decider" and its decision making rules may be based on probabilistic data, for example. Figure 3 also shows the valve 1 component commanded by the $\mathrm{OH}$ valve 1 ; the PFS and e-PN models of valve 1 model considering the influence of the control 
signal transmission network; the diagnoser for the valve 1 and flowmeter; and the related decider device. In Fig. 4 is presented an example of fault treatment and its reconfiguration (degeneration).

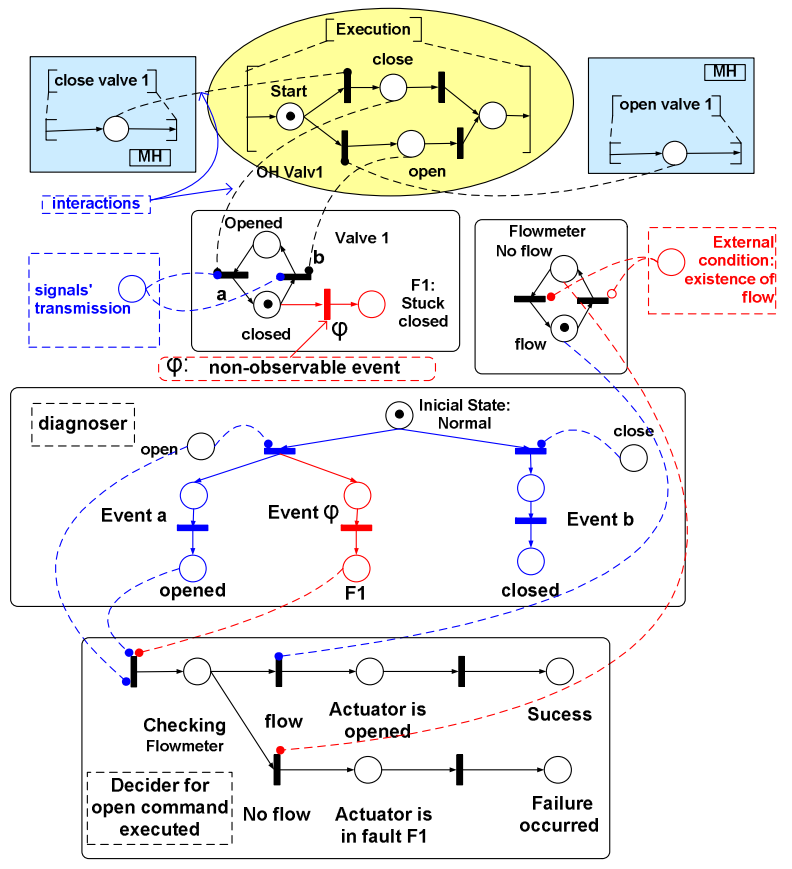

Fig. 3. Example of interactions, and control objects models, diagnoser and decider
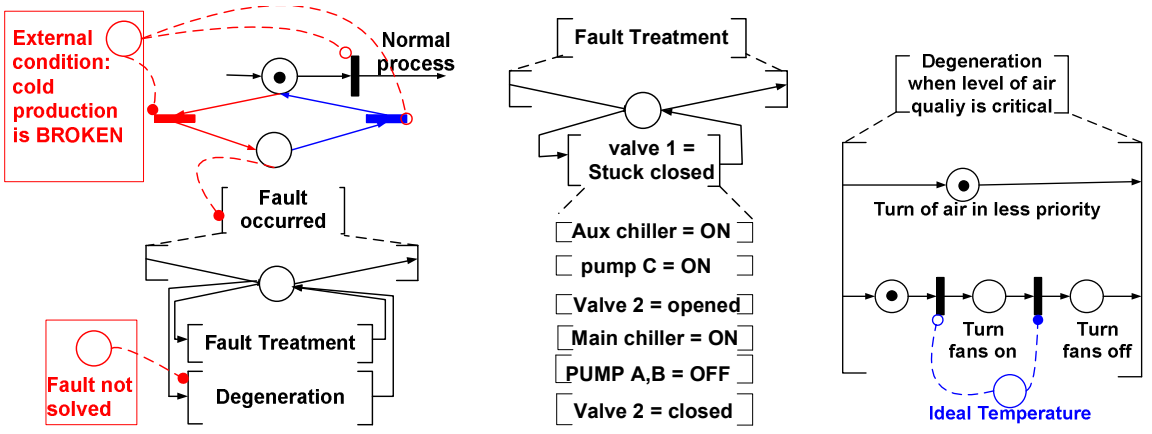

Fig. 4. Example of fault treatment and its reconfiguration (degeneration)

Phase 3 - analysis/ simulation - the analysis is developed with e-PN tools for edition and structural analysis. The behavior and the quantitative analysis were carried out by means of associated simulation techniques with checking of e-PN properties. This type of analysis allows re-design and re-engineering of the control system during the design phase. This phase is subdivided in: qualitative and 
quantitative analysis. Qualitative analysis allows the verification of structural properties and behavioral models, sketching conclusions about the system operation, such as: i) liveness, that is related to the complete absence of deadlocks in operating systems; ii) reachability, to study the dynamic properties of any system; iv) reversibility, to recover from disruptive events of the operation; v) conservation and boundedness to verify the variation of the number of tokens of the net. The quantitative analysis requires the introduction of the time parameter associated with the transitions. Thus, it is possible check if the firing is consistent with specifications of the models.

Phase 4 - implementation - for the practical use, the resulting models are interpreted as control program specifications to be performed by computers (supervisory control) and programmable controllers (local control level). This phase also comprises the codification, parameterization and development of wrapper interfaces.

Phase 5 - operation - in this phase, the real-time supervision of the automation control system is performed by synchronizing the operation of the AHFTCS with the e-PN models, in order to control and monitor the system. The signals from the sensors and the status of mechatronic devices are acquired and connected with e-PN models. The adaptation and re-configuration of the FPS is supported using this procedure, i.e., the introduction or remotion of new components requires the addition or remotion of a new token in the corresponding e-PN models and, in some cases, the modification of associated holons models.

\section{Conclusions and Future Works}

Using as application example an intelligent building (IB), a novel procedure for design of AHFTCS considering normal operations and occurrence of faults in flexible productive system (FPS) was presented. The process combines the requirements of the holonic control system (HCS) and AFTCS (active fault-tolerant control system), with special attention to the system's reconfiguration. The modeling process is based on interpreted Petri net (PN), and its extension called PFS is used to structure the development of components' models and presentation of the proposed procedure, combining the bottom-up approach and the top-down approach of the stepwise refinement associated to PFS. The use of this systematic technique, to structure and rationalize the models development of the proposed architecture allows an environment that facilitates the development of new models. The proposed architecture and its mechanisms allow implementing a hierarchic or heterarchic control structure and reacts to faults more agilely. This work synthesizes Silva's project [3], which involved modeling of the whole HVAC and other subsystems of IB such as: access control, fire fight and prevention, people transportation/ movement, and signals transmission control; besides the simulation and validation of extended Petri net (e-PN) models.

The $\mathrm{PhD}$ thesis of one of the authors, the student Silva, involves the whole life cycle of automation systems. Since the research done so far does not yet offers a complete solution to extending the research results towards applicability in other supervision and control systems. More detailed case studies for a complete evaluation are needed. The survey of theories, tools and applications, are considered as the most feasible and adequate research strategy in this study. The next stage of research 
involves the transformation of a conceptual model, which must be developed and refined by the general surveys, to a practical model.

\section{References}

1. Schoop, R., Colombo, A.W., Suessmann, B., Neubert, R.: Industrial experiences, trends and future requirements on agent-based intelligent automation. In: Proceedings of IECON the Annual Conference of IEEE Industrial Electronics Society, Seville (2002)

2. Leitão, P., Colombo, A.W.: Petri net based Methodology for the Development of Collaborative Production Systems. In: Proceedings of the 11th IEEE International Conference on Emerging Technologies and Factory Automation (ETFA 2006), Prague, pp. 819-826 (2006)

3. Silva, R.M., Arakaki, J., Miyagi, P.E., Junqueira, F., Santos Filho, D.J.: Intelligent Building - Modeling and Reconguration using Petri net and Holons. In: Proceedings of ICNPAA: the 8th IEEE Int. Conf. on Mathematical Problems in Engineering, Aerospace and Sciences, São José dos Campos, Brazil (2010)

4. Zhang, Y., Jiang, J.: Bibliographical review on reconfigurable fault-tolerant control systems. Annual Reviews in Control 32, 229-252 (2008)

5. Arakaki, J., Miyagi, P.E.: Degeneration methods in intelligent building control system design, Boston. IFIP, vol. 220, pp. 469-478 (2006)

6. Sousa, P., Ramos, C., Neves, J.: The Fabricare System. Production Planning \& Control 15(2), 156-165 (2004)

7. Colombo, A.W., Neubert, R., Schoop, R.: A solution to holonic control systems. In: Proceedings of ETFA the 8th IEEE Int. Conf on Emerging Technologies and Factory Automation, Sophia/Nice (2001)

8. Wong, J.K.W., Li, H.: Construction, application and validation of selection evaluation model (SEM) for intelligent HVAC control system. Automation in Construction 19, 261-269 (2010)

9. Zickler, A., Mennicken, L.: Science for Sustainability: The Potential for German-Brazilian Cooperation on sustainability-oriented Research and Innovation - Introduction. In: Proceedings of the 1st German-Brazilian Conference on Research for Sustainability, São Paulo, Brazil (2009)

10. Blackstocka, K.L., Kellyb, G.J., Horseyb, B.L.: Developing and applying a framework to evaluate participatory research for sustainability. Ecological Economics (60), 726-742 (2007)

11. David, R., Alla, H.: Petri nets for modeling of dynamic systems - a survey. Automatica 30(2), 175-201 (1994)

12. Hasegawa, K., Miyagi, P.E., Santos Filho, D.J., Takahashi, K., Ma, L.Q., Sugisawa, M.: On resource arcs for Petri net modeling of complex shared resource systems. Journal of Intelligent \& Robotic Systems 26(3/4), 423-437 (1999)

13. Sampath, M., Sengupta, R., Lafortune, S., Sinnamhoideen, K., Teneketzis, D.C.: Failure diagnosis using discrete-event models. IEEE Trans. on Control Systems Technology 4(2), 105-124 (1996)

14. Kuipers, B.: Qualitative Reasoning: Modeling and Simulation with Incomplete Knowledge. The MIT Press, Cambridge (1994)

15. Scheidt, D.H.: Intelligent Agent-Based Control. Johns Hopkins APL Technical Digest 23(4), 383-395 (2002)

16. Booch, G., Rumbauch, J., Jacobson, I.: The Unified Modeling Language User Guide. Addison Wesley Longman, Inc., Amsterdam (1999) 